\title{
EL DESARROLLO DE LA MARCHA INFANTIL COMO PROCESO DE APRENDIZAJE CHILDREN GAIT DEVELOPMENT AS A LEARNING PROCESS
}

\author{
Patricia Martín Casas ${ }^{a^{*}}$, Alfonso Meneses Monroy ${ }^{b}$, Juan Vicente Beneit \\ Montesinos $^{c}$ Y M. . Ángeles Atín Arratibel $^{\mathrm{a}}$
}

Cómo referenciar este artículo/How to reference this article:

Martín Casas, P., Meneses Monroy, A., Beneit Montesinos, J. V. y Atín Arratibel, M. A. (2014). El Desarrollo de la Marcha Infantil como Proceso de Aprendizaje [Children Gait Development as a Learning Process]. Acción Psicológica, 11(1), 45-54. http://dx.doi.org/10.5944/ap.1.1.13866

\section{Resumen}

El desarrollo de la marcha infantil es un proceso especialmente complejo en que el intervienen factores motores, perceptivos y cognitivos. Desde el nacimiento, el niño integra la información recibida y realiza un progresivo control cortical sobre los patrones neuromotores innatos para desarrollar diferentes formas de locomoción hasta la marcha independiente. Estas ideas no son novedosas pero se están revisando en la actualidad y han motivado numerosos cambios en el estudio del desarrollo infantil. La consideración de la adquisición de la marcha como un aprendizaje en situaciones de constante cambio permite comprender la marcha como acción, cuya realización implica la adquisición de nuevas capaci- dades de interacción y conocimientos. En este trabajo se exponen los principales conocimientos acerca de este proceso de aprendizaje y se propone destacar las alteraciones de la marcha como indicadores de dificultades en el desarrollo, que requieren una valoración y un abordaje terapéutico interdisciplinares.

Palabras clave: Marcha; niños; desarrollo; valoración.

\begin{abstract}
Children gait development is a particularly complex process in which motor, perceptual, and cognitive factors interact. From the moment of birth, the child integrates the information he receives and builds up a progressive
\end{abstract}

a Departamento de Medicina Física y Rehabilitación. Hidrología Médica. Facultad de Enfermería, Fisioterapia. Universidad complutense de Madrid.

b Escuela de Enfermería de la Cruz Roja Española. Universidad Autónoma de Madrid.

c Departamento de Enfermería. Facultad de Enfermería, Fisioterapia y Pología. Universidad Complutense de Madrid.

* Autor de correspondencia E-mail: pmcasas@enf.ucm.es. Tel. 913947274. 
cortical control on the innate neuromotor patterns to develop different forms of locomotion until achieving independent gait. These ideas are not new but they are currently being reviewed and have encouraged numerous changes in the study of child development. Considering gait acquisition as a learning process in changing situations allows to understand gait as an action which involves the acquisition of new knowledge and interaction capabilities. This paper summarizes the main body of knowledge about this learning process and intends to highlight gait alterations as indicators of development difficulties, requiring interdisciplinary assessment and therapeutic approach.

Keywords: Gait; children; development; assessment.

\section{Introducción: Complejidad del patrón de marcha e implicaciones de su evaluación}

La marcha es una actividad altamente compleja por la dificultad para mantener el equilibrio que implica, lo cual requiere la consideración de una gran cantidad de informaciones relativas al propio cuerpo y al ambiente. Además, estas informaciones son cambiantes y deben ponerse en relación con las de referencia para cada persona, a fin de resultar útiles para el control motor (Adolph, 2008; ShumwayCook y Woollacott, 2007).

Los sistemas motor y perceptivo se desarrollan en los niños de forma interdependiente, a través de la exploración. De este modo, las diversas estructuras implicadas en el control motor, tanto centrales como periféricas, experimentan un progresivo desarrollo y una integración funcional que da lugar a comportamientos cada vez más complejos y coordinados (Bril y Ledebt, 1998; Zwart, Ledebt, Fong, De Vries y Savelsbergh, 2005). La alteración de uno de estos sistemas o de ambos y la consecuente formación deficitaria del esquema corporal podrían resultar factores limitantes para el desarrollo de un patrón de marcha correcto y adaptable (Assaiante y Amblard,
1992; Dal Molin, 1994; Metcalfe y Clark, 2000). Por este motivo, es imprescindible llevar a cabo en la valoración infantil una serie exploraciones que permitan conocer en cada niño el desarrollo de las diferentes habilidades perceptivas, cognitivas y motoras, ligadas al proceso de maduración progresiva del sistema nervioso central (Portellano, 2007). Asimismo, resulta necesario conocer la elaboración individual de los diversos procesos a partir de las diversas experiencias vividas desde el nacimiento, dada la importancia vital de la experiencia en el desarrollo infantil (Adolph, Vereijken y Shrout, 2003; Joh y Adolph, 2006; Thelen, Schöner, Scheier y Smith, 2001; Zelazo, 1983).

Este trabajo tiene como objetivo exponer las diferentes teorías sobre el desarrollo de la marcha infantil, que desde una perspectiva histórica y también neurofisiológica pueden subdividirse en tres corrientes claramente diferenciadas. A través de su lectura, el lector podrá comprender cómo desde una concepción puramente neuromotora se han integrado conocimientos y experiencias que conducen a considerar la marcha como una actividad de aprendizaje en situaciones de constante cambio. Por ello, resulta un excelente indicador del desarrollo de los sistemas perceptivos, cognitivo y motor, con influencia también de aspectos emocionales y sociales (Adolph, 2008; Berger y Adolph, 2007). Las aportaciones recogidas en el presente trabajo invitan a destacar la importancia de evaluar en todos los niños el patrón de marcha como indicador de posibles dificultades en el desarrollo, para lo cual resulta fundamental conocer los procesos subyacentes a la organización y ejecución de la marcha y sus posibles modalidades, que se exponen a continuación.

\section{El desarrollo de la marcha infantil, desde los patrones reflejos al control voluntario}

Desde el nacimiento del niño, cuando empieza a patalear, comienza un período de unos doce meses en el que estos movimientos, aparentemente indiferenciados, aleatorios y sin objetivo, se transforman para permitir la apa- 
rición de la marcha bípeda, que no dejará de modificarse a lo largo de toda la vida (Gesell, 1997; Leonard, 1990). Aunque existen otras teorías que lo explican exclusivamente desde un punto de vista madurativo, los conocimientos actuales apoyan que el desarrollo de la marcha se considere desde la perspectiva de los sistemas dinámicos enunciada por Thelen et al., según la cual cada tarea debe estudiarse desde un enfoque integral basado en el bucle percepción-acción, que permite adecuar el comportamiento a las características propias y del entorno en cada momento concreto. Esta aproximación facilita la comprensión del proceso global de desarrollo y del niño, de sus alteraciones y de la actuación terapéutica desde cualquier disciplina (Kamm, Thelen y Jensen, 1990; Leonard, 1990; Thelen, Kelso y Fogel, 1987; Williams, Tinley y Curtin, 2010).

El desarrollo de las habilidades motoras bajo control voluntario, organizado por los centros superiores de control, ha sido ampliamente investigado por diversos autores. Zelazo y cols. indicaron que los llamados reflejos primitivos son patrones motores innatos y automáticos, que pueden ser modificados mediante el aprendizaje instrumental adquirido con la experiencia para convertirse en respuestas controladas voluntariamente. De acuerdo con este modelo, de-reflejo-a-instrumental, las transiciones en el comportamiento motor estarían en relación directa con cambios en el procesamiento central. Por ello, la maduración cognitiva y la capacidad para procesar información pueden ser factores limitantes para el logro de la marcha bípeda independiente (Zelazo, 1983). En este proceso, algunas competencias motoras reflejas del neonato humano, tales como la reacción de sostén o de la de dar pasos con apoyo, se consideran respuestas estereotipadas que permanecen en el repertorio de movimientos del niño hasta aproximadamente el final del tercer mes, cuando tradicionalmente se piensa que desaparecen. Esta desaparición de los patrones reflejos ha sido atribuida a la inhibición producida por la maduración de centros corticales superiores, aunque podrían estar también implicados otros factores como el incremento de masa corporal y el enlentecimiento en la adaptación neuromuscular ante este cambio (Leonard, 1990; Thelen y Cooke, 1987). Desde el punto de vista de la teoría de los sistemas dinámicos, se puede afirmar que el desarrollo de habilidades locomotoras es el resultado de un fenómeno multidimensional de integración progresiva de las distintas capacidades perceptivas y motoras (Brenière y Bril, 1998; Thelen, Kelso et al., 1987).

Hacia el año de edad, aproximadamente, aparecen cambios en la función motora junto con cambios en la habilidad de procesar la información, lo que origina que en un corto período de tiempo se produzcan profundas modificaciones en el desarrollo motor, cognitivo y lingüístico. Alrededor de los doce meses de vida, los niños dan sus primeros pasos independientes, dicen sus primeras palabras y comienzan a usar los objetos en una actividad dirigida a un objetivo. Zelazo propuso que la base para estos cambios se encuentra en una transformación en la habilidad para procesar la información, que permite que los niños puedan crear asociaciones y recuperar información almacenada con rapidez, evolución que resulta particularmente significativa para el desarrollo motor (Zelazo, 1983). De este modo, los cambios en la capacidad de procesamiento central permiten la integración de habilidades espaciales, perceptivas y de coordinación que favorecen la transición de la locomoción cuadrúpeda a la bípeda (Zelazo y Leonard, 1983).

Durante este proceso, los patrones motores innatos resultan modificados desde una respuesta estereotipada subcortical hasta una respuesta regulada por un centro superior de control. En lugar de desaparecer, actualmente se considera que estos patrones innatos son inhibidos o controlados por los centros superiores mientras que constituyen la base para el comportamiento motor voluntario posterior, en una transformación que sucede gradualmente durante la infancia y la niñez mediante práctica y experiencia, existiendo por tanto una continuidad en la función motora (Figura 1). Así, se ha descartado que las formas innatas y posteriores del mismo comportamiento motor no estén relacionadas y parece que el aprendizaje instrumental puede explicar la modulación del con- 
trol motor (Shumway-Cook y Woollacott, 2007; Zelazo, 1983). Forssberg validó este modelo ya en los años 80, mediante la confirmación del mantenimiento electromiográfico del patrón desde la marcha automática neonatal hasta la madura, diferenciándose ambas en que el primer patrón parece ser iniciado por un estiramiento sobre la cadera cuando el niño es inclinado hacia delante para iniciar la marcha, el niño necesita soporte externo para permanecer erecto y la marcha se realiza con el apoyo de la punta del pie y no de toda la planta (Forssberg, 1985). Además, durante la marcha madura la flexión de cadera y rodilla están coordinadas mientras que en el tobillo se produce una fase inversa, que permite el desarrollo del choque de talón durante la marcha. Sin embargo, estas diferencias no excluyen la continuidad del patrón, ya que la marcha automática, la locomoción con apoyo y la marcha independiente en los niños comparten componentes constituyentes, con una progresión de desarrollo. Esta evolución sugiere que los cambios graduales en la organización del paso se producen a partir de las demandas dinámicas funcionales de la locomoción bípeda, junto con el desarrollo del equilibrio, el control postural y la fuerza durante el primer año de vida (Cooke y Thelen, 1987; Thelen y Cooke, 1987).

\section{Desarrollo del control central de la marcha}

A partir de sus estudios, Forssberg postuló una teoría de control neural para explicar los cambios en el desarrollo de la marcha infantil. Propuso que las redes neuronales innatas, posiblemente controladas a nivel medular, generan patrones locomotores y mientras estos patrones cambian con la maduración del mecanismo central de control, el mismo circuito neural se utiliza para la locomoción del recién nacido y para la marcha madura (Forssberg, 1985). Otros autores también apoyaron la idea de un programa central motor que genera un patrón de movimiento caracterizado temporal y espacialmente, pues los patrones de pataleo y de marcha constituyen una respuesta isomórfica con una precisa coordinación entre extremidades (Clark, Whitall y
Phillips, 1988; Ivanenko, Dominici, Cappellini y Lacquaniti, 2005; Schneider, Zernicke, Ulrich, Jensen y Thelen, 1990; Thelen, Ulrich y Niles, 1987; Yang et al., 2004). Pero sobre este patrón de base, desde el comienzo de la marcha los niños varían su estrategia en función de sus propias características y las del entorno, lo que refleja una capacidad emergente para la organización dinámica (Clark et al., 1988; Lamb y Yang, 2000; Thelen y Cooke, 1987; Yang et al., 2004).

A su vez, la regulación de la postura y el equilibrio parece depender de un centro superior de control motor que se desarrolla durante la infancia, el cual puede relacionarse con la marcha activada espinalmente hacia el final del primer año de vida (Leonard, 1990). Forssberg ya propuso que el desarrollo de la marcha independiente depende de la maduración del sistema de equilibrio y no se realiza exclusivamente por los cambios en el patrón locomotor, pues observó que los niños no parecen realizar voluntariamente la marcha hasta que el equilibrio se desarrolla adecuadamente para mantener el control postural antigravitatorio (Forssberg, 1985). Al analizar las estructuras más implicadas en este control del equilibrio, algunos autores abogan por atribuir la mayor importancia en este proceso al tronco, junto con la cabeza y la pelvis, pues lo consideran un marco inicial de referencia fundamental para el control de la orientación y el equilibrio. Desde esta hipótesis, el desarrollo del control postural se produce desde la creación de referencias a partir del propio cuerpo (sistema egocéntrico) hasta la consideración prioritaria de los elementos del entorno (sistema exocéntrico) (Assaiante, Mallau, Viel, Jover y Schmitz, 2005). No obstante, no se puede considerar el control postural del tronco como independiente a los patrones de movimiento, sino que parece existir una interrelación bidireccional entre el control postural y la actividad locomotora, como demuestra la variación de los patrones de pataleo en las diferentes posturas y que los niveles de desarrollo locomotor en niños resultan mejores predictores del control del equilibrio estático que la propia edad (Jensen, Schneider, Ulrich, Zernicke y Thelen, 1994; Sundermier, Woollacott, Roncesvalles y Jensen, 2001). 
Se puede considerar entonces que el desarrollo de la marcha corresponde a un proceso de aprendizaje progresivo para producir y modular fuerzas de propulsión y afrontar situaciones de desequilibrio dinámico durante el acto de caminar (Bril y Brenière, 1992). Este aprendizaje, estrechamente relacionado con la experiencia, puede realizarse a partir del progresivo control descendente, teoría demostrada por algunos de los estudios ya mencionados y también por la progresiva disminución en los niños de la variabilidad de la posición del pie durante la fase de balanceo al caminar en una cinta de marcha, relacionada con la maduración del control corticoespinal de los músculos dorsiflexores del pie (Petersen, Kliim-Due, Farmer y Nielsen, 2010). En relación con estos conceptos, también el desarrollo de una marcha con choque de talón a partir de la marcha con el apoyo preferente de la punta del pie se considera el resultado de adaptaciones posturales y motoras a partir del desarrollo central (Forssberg, 1985; Kamm et al., 1990).

En cuanto a la evolución de este control central, Bril y Brenière establecieron un proceso de adquisición de la marcha en dos fases; la primera corresponde al aprendizaje de los requerimientos posturales de la marcha, de 3 a 6 meses después del comienzo de la locomoción independiente, y la segunda, que dura varios años, está destinada a un fino ajuste de la marcha que se estabiliza hacia los 7-8 años, edad en la que el control del desplazamiento del centro de gravedad y el patrón de activación muscular adulto son completos (Bril y Brenière, 1992; Bril y Ledebt, 1998; Cavagna, Franzetti y Fuchimoto, 1983; Ledebt, Bril y WienerVacher, 1995). Posteriormente las mismas autoras determinaron que la capacidad postural necesaria para controlar el equilibrio con la musculatura de los miembros inferiores no se consigue antes de los 4-5 años de marcha independiente, es decir, a los 5-6 años de edad
(Brenière y Bril, 1998). Sin embargo, el control de la cabeza, la integración de los parámetros corporales y el desarrollo de ajustes posturales anticipatorios necesitan de al menos 8 años de experiencia de marcha para desarrollarse $\mathrm{y}$, además, la experiencia de actividades de locomoción tiene un papel determinante en el control del movimiento de los miembros inferiores y del equilibrio hasta, al menos, los 10 años de edad (Assaiante y Amblard, 1996; Hirschfeld y Forssberg, 1992; Ledebt y Brenière, 1994; Ledebt, Bril y Brenière, 1998; Roncesvalles, Woollacott y Jensen, 2001). En este proceso largo y progresivo, el control postural antigravitatorio, el equilibrio y la coordinación de movimientos requieren una considerable integración de los componentes sensoriomotores junto con la intención motivacional para conducir el movimiento, con las funciones superiores gobernando dicha respuesta (Clark et al., 1988; Leonard, 1990).

\section{Integración entre percepción y acción}

Como se ha expuesto anteriormente, el desarrollo motor temprano se produce a partir de una organización prefuncional en el sistema nervioso central del niño para el movimiento, mediante patrones neuromotores elementales, con un alto grado de estereotipia, que se activan bajo condiciones específicas en todos los niños. Estos patrones elementales representan movimientos complejos primordiales que anuncian la posterior aparición de actividades más desarrolladas, como arrastrarse, gatear, sentarse y caminar (Figura 1). De este modo, los patrones neuromotores innatos están organizados ya desde antes del nacimiento, proporcionan un sustrato a partir del cual se desarrollan los movimientos controlados y favorecen la ejecución de patrones de movimientos rítmicos, tanto en el tiempo como en el espacio. 


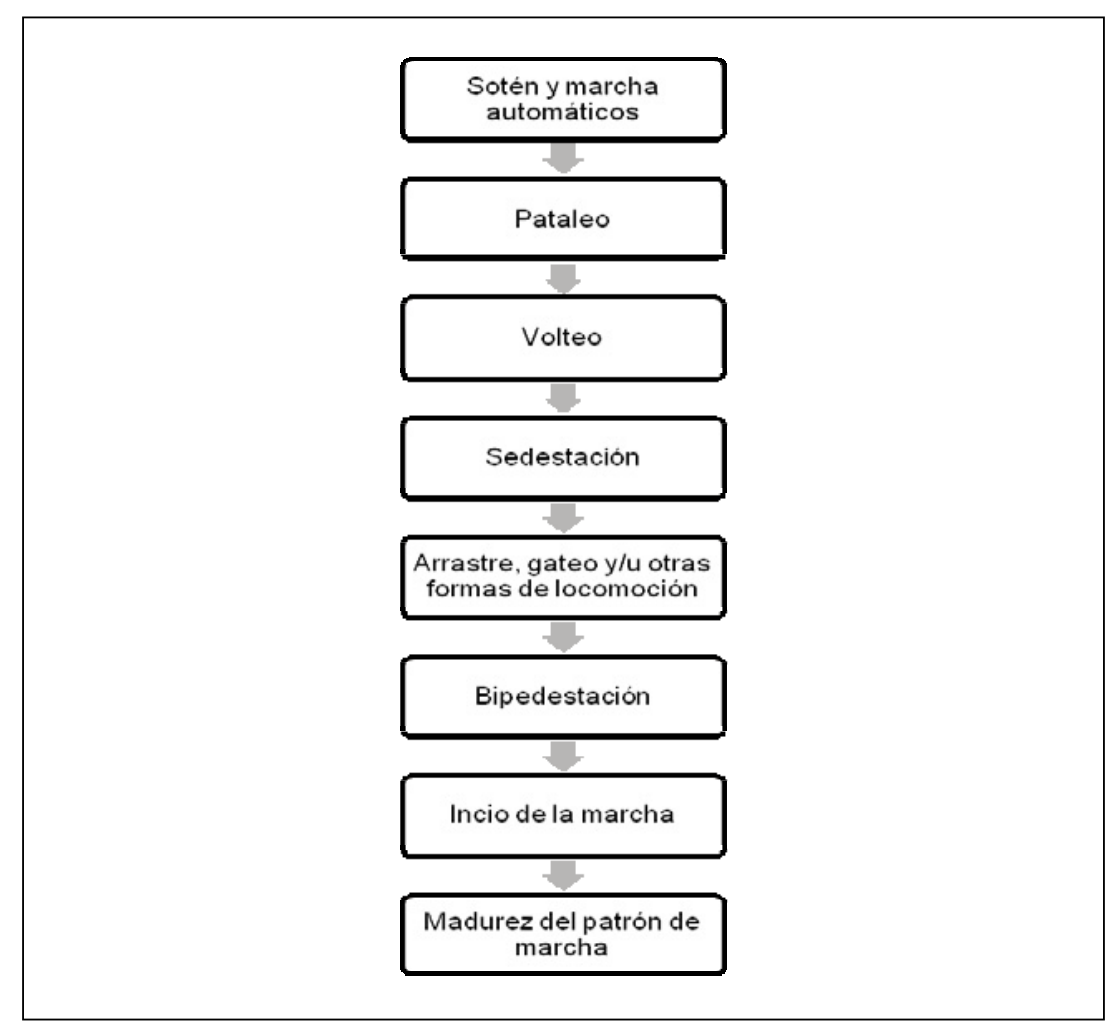

Figura 1. Proceso de desarrollo de la marcha infantil.

Hitos del desarrollo sensoriomotor relacionados con la marcha

Pero aunque estos patrones primitivos marquen el comienzo de algunos actos motores, en conjunto, el desarrollo motor depende de otros factores tales como la biología, el comportamiento y el entorno del niño, pues éste responde en cada momento a las demandas y características del exterior y del propio cuerpo, combinadas entre sí (Adolph y Avolio, 2000; Angulo-Kinzler, Ulrich y Thelen, 2002; Fogel y Thelen, 1987; Kamm et al., 1990; Leonard, 1990). Existe constantemente una inte- racción recíproca entre las características del entorno y sus demandas, las necesidades del niño y el desarrollo sensoriomotor, que propicia la evolución constante del comportamiento a partir de acontecimientos externos o generados por el propio niño (Figura 2). Por ejemplo, se ha referido que las informaciones recibidas al asumir una postura vertical podrían fomentar el interés del niño en eventos distantes y proporcionarle la motivación para dar sus primeros pasos independientes (Zelazo, 1983). 


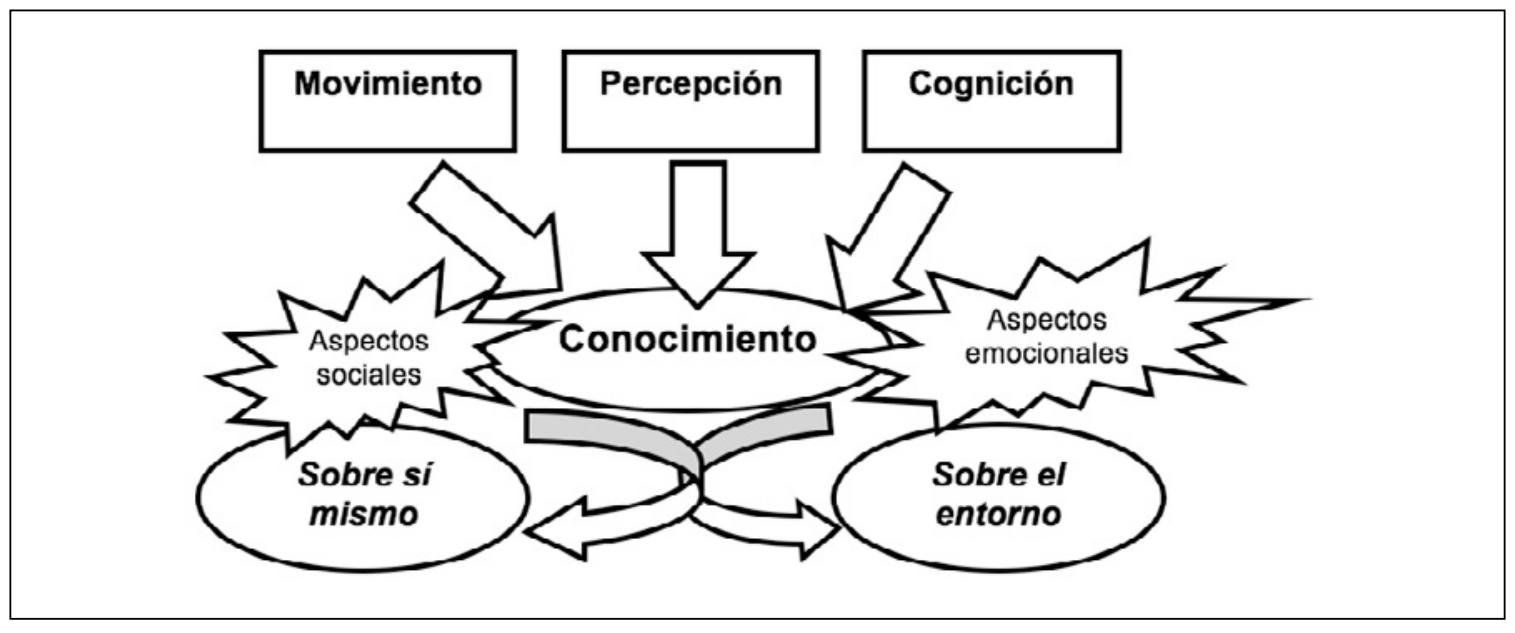

Figura 2. Marcha como proceso de aprendizaje. Integración entre percepción, cognición y movimiento durante la marcha infantil, que permite a su vez la recogida de informaciones y la adquisición de conocimientos sobre uno mismo y sobre el medio externo (los objetos y las personas), base para la construcción de aspectos emocionales y sociales

Desde mediados de la década de los 80 , el estudio de la adquisición de las habilidades motoras ha experimentado un renacimiento bajo una nueva corriente que enfatiza la naturaleza multicausal, fluida, contextual y auto-organizada del cambio del desarrollo, la unidad de la percepción, acción y cognición y el papel de la exploración y selección de informaciones y estrategias en la aparición de nuevos comportamientos (Thelen, 1995). A la vez que la transición a la marcha independiente produce cambios significativos en la forma en que los niños se relacionan con su entorno, este hito del desarrollo se concibe como un proceso de integración durante el cual el niño tiene que aprender a ajustar las sinergias posturales para mantener el equilibrio en las fases dinámicas de apoyo mono y bipodal (Bril y Ledebt, 1998; Clearfield, Osborne y Mullen, 2008). Para ello, todas las aferencias que recibe han de diferenciarse y seleccionarse previamente, especialmente en situaciones de conflicto sensorial en las que la información no es congruente, proceso en el que resulta fundamental el desarrollo de cada modalidad, pues los cambios en uno o más de los sistemas sensoriales podrían subyacer a los cambios comportamentales que ocurren durante el desarrollo motor (Bril y Ledebt,
1998). A partir de estos presupuestos teóricos, se ha demostrado que diversas informaciones sensoriales pueden generar desde etapas tempranas los cambios en la estrategia motora, condicionados por las variaciones del entorno y del propio cuerpo. La exploración permite el control prospectivo de la marcha desde los primeros pasos, aunque su control adaptativo requiere de un largo proceso de aprendizaje de la relación entre la información percibida y las consecuencias para el control del equilibrio (Adolph, 2008; Adolph y Avolio, 2000; Adolph, Eppler, Marin, Weise y Wechsler, 2000; Berger y Adolph, 2007; Joh y Adolph, 2006; Lam, Wolstenholme y Yang, 2003). Este proceso de aprendizaje puede estar limitado por la capacidad cognitiva (Leonard, 1990; Zelazo, 1983), además de por un déficit en la recogida de una o varias modalidades sensoriales o la dificultad en su integración (Williams et al., 2010).

En conjunto, durante el desarrollo infantil se produce un progresivo control de los distintos sistemas implicados en la marcha, que promueve una evolución del patrón de marcha en el que la experiencia ha demostrado ser el factor con mayor influencia, lo que apoya la teoría del aprendizaje instrumental (Adolph et al., 2003; Joh y Adolph, 2006; Zelazo, 1983). 
En la actualidad, este proceso de elaboración y aprendizaje a partir de la experiencia se considera la base del denominado control motor, que requiere la integración de todas las informaciones relevantes (Figura 2) (ShumwayCook y Woollacott, 2007; Williams et al., 2010). En este proceso, tienen además un importante papel la motivación, la percepción del riesgo y, en general, los procesos corticales superiores, con una relación recíproca entre la locomoción adaptativa y la cognición (Adolph, Tamis-LeMonda, Ishak, Karasik y Lobo, 2008; Cherng, Liang, Chen y Chen, 2009). Se ha demostrado que los límites y las ventajas para resolver problemas y la capacidad perceptiva y cognitiva afectan a la habilidad de los niños para moverse en un entorno cambiante, mientras que el desarrollo locomotor les ofrece nuevas oportunidades de aprendizaje sobre sí mismos, el medio que les rodea y la relación entre ambos (Berger y Adolph, 2007).

\section{Conclusiones}

La influencia de los procesos sensoriomotores y de integración en la organización y ejecución de la marcha resulta evidente en los procesos de desarrollo normal y también en numerosas alteraciones de la marcha infantil, tales como la marcha de puntillas idiopática. Esta perspectiva global del desarrollo infantil puede facilitar una mejor comprensión de dichas alteraciones e impulsar un abordaje terapéutico precoz, pues las desviaciones de la normalidad en el proceso de adquisición y evolución de la marcha deben considerarse un indicador que sugiere la necesidad de una valoración exhaustiva e interdisciplinar del desarrollo. En ella, habrían de considerarse no solamente los aspectos motores, sino también los perceptivos y cognitivos. A partir de estas aportaciones, resulta fundamental que todos los profesionales que trabajan con niños consideren la globalidad del desarrollo infantil e interpreten las diversas alteraciones desde una perspectiva integral, para proporcionar las indicaciones y el tratamiento óptimos. No obstante, son necesarios más estudios para profundizar en este tema y fomentar la colaboración interdisciplinar en el abordaje de las alteraciones infantiles.

\section{Referencias bibliográficas}

Adolph, K. E. (2008). Learning to move. Current Directions in Psychological Science, 17(3), 213-218.

Adolph, K. E. y Avolio, A. M. (2000). Walking infants adapt locomotion to changing body dimensions. Journal of Experimental Psychology: Human Perception and Performance, 26(3), 1148-1166.

Adolph, K. E., Eppler, M. A., Marin, L., Weise, I. B. y Wechsler, M. (2000). Exploration in the service of prospective control. Infant Behavior and Development, 23, 441-460.

Adolph, K. E., Tamis-LeMonda, C. S., Ishak, S., Karasik, L. B. y Lobo, S. A. (2008). Locomotor experience and use of social information are posture specific. Developmental Psychology, 44(6), 1705-1714.

Adolph, K. E., Vereijken, B. y Shrout, P. E. (2003). What changes in infant walking and why. Child Development, 74(2), 475-497.

Angulo-Kinzler, R. M., Ulrich, B. y Thelen, E. (2002). Three-month-old infants can select specific leg motor solutions. Motor Control, 6(1), 52-68.

Assaiante, C. y Amblard, B. (1992). Peripheral vision and age-related differences in dynamic balance. Human Movement Science, 11, 533-548.

Assaiante, C. y Amblard, B. (1996). Visual factors in the child gait: effects on locomotor skills. Perceptual and Motor Skills, 83, 1019-1041.

Assaiante, C., Mallau, S., Viel, S., Jover, M. y Schmitz, C. (2005). Development of postural control in healthy children: a functional approach. $\mathrm{Neu}$ ral Plasticity, 12(2-3), 109-118.

Berger, S. E. y Adolph, K. E. (2007). Learning and development in infant locomotion. Progress in Brain Research, 164, 237-255.

Brenière, Y. y Bril, B. (1998). Development of postural control of gravity forces in children during the first 5 years of walking. Experimental Brain Research, 121, 255-262.

Bril, B. y Brenière, Y. (1992). Postural requirements and progression velocity in young walkers. Journal of Motor Behavior, 24, 105-116.

Bril, B. y Ledebt, A. (1998). Head coordination as a means to assist sensory integration in learning 
to walk. Neurosci Biobehav Rev Neuroscience \& Biobehavioral Reviews, 22(4), 555-563.

Cavagna, G. A., Franzetti, P. y Fuchimoto, T. (1983). The mechanics of walking in children. Journal of Physiology 343, 323-339.

Clark, J. E., Whitall, J. y Phillips, S. J. (1988). Human interlimb coordination: The first 6 months of independent walking. Developmental Psychobiology, 21, 445-456.

Clearfield, M. W., Osborne, C. N. y Mullen, M. (2008). Learning by looking: Infants' social looking behavior across the transition from crawling to walking. Journal of Experimental Child Psychology, 100, 297-307.

Cooke, D. W. y Thelen, E. (1987). Newborn stepping: A review of puzzling infant co-ordination. Developmental Medicine \& Child Neurology, 29(3), 399-404.

Cherng, R. J., Liang, L. Y., Chen, Y. J. y Chen, J. Y. (2009). The effects of a motor and a cognitive concurrent task on walking in children with developmental coordination disorder. Gait \& Posture, 29, 204-207.

Dal Molin, G. (1994). Il cammino: studiarlo per poterlo rieducare [Gait: study to be able to reeducate]. Riabilitazione e apprendimento, 3/4, 153-162.

Fogel, A. y Thelen, E. (1987). Development of early expressive and communicative action. Reinterupting the evidence from a dynamic systems perspective. Child Development, 23, 747.

Forssberg, H. (1985). Ontogeny of human locomotor control. I. Infant stepping, supported locomotion, and transition to independent locomotion. Experimental Brain Research, 57, 480-493.

Hirschfeld, H. y Forssberg, H. (1992). Development of anticipatory postural adjustements during locomotion in children. Journal of Neurophysiology, 68, 542-549.

Ivanenko, Y. P., Dominici, N., Cappellini, G. y Lacquaniti, F. (2005). Kinematics in newly walking toddlers does no depend upon postural stability. Journal of Neurophysiology, 94, 754-763.

Jensen, J. L., Schneider, K., Ulrich, B. D., Zernicke, R. F. y Thelen, E. (1994). Adaptative dynamics of the leg movement patterns of human infants:
I. The effects of posture on spontaneous kicking. Journal of Motor Behavior, 26(4), 303-312.

Joh, A. S. y Adolph, K. E. (2006). Learning from falling. Child Dev, 77(1), 89-102.

Kamm, K., Thelen, E. y Jensen, J. L. (1990). A dinamical systems approach to motor development. Physical Therapy, 70, 763-775.

Lam, T., Wolstenholme, C. y Yang, J. F. (2003). How do infants adapt to loading of the limb during the swing phase of stepping? Journal of Neurophysiology, 89, 1920-1928.

Lamb, T. y Yang, J. F. (2000). Could differents directions of infant stepping be controlled by the same locomotor central pattern generator? Journal of Neurophysiology, 83, 2814-2824.

Ledebt, A. y Brenière, Y. (1994). Dynamic implications of anatomical and mechanical parameters in gait iniciation process in children. Human Movement Science, 13, 801-815.

Ledebt, A., Bril, B. y Brenière, Y. (1998). The buildup of anticipatory behaviour. An analysis of the development of gait iniciation in children. Experimental Brain Research, 120(1), 9-17.

Ledebt, A., Bril, B. y Wiener-Vacher, S. (1995). Trunk and head stabilization during the first months of independent walking. NeuroReport, 6, 1737-1740.

Leonard, E. L. (1990). Early motor development and control: Foundations for independent walking. In G. L. Smith (Ed.), Gait in Rehabilitation. Clinics in Physical Therapy (pp. 121-140). New York: Churchill Livingstone.

Metcalfe, J. S. y Clark, J. E. (2000). Sensory information affords exploration of posture in newly walking infants and toddlers. Infant Behavior \& Development, 23, 391-405.

Petersen, T. H., Kliim-Due, M., Farmer, S. F. y Nielsen, J. B. (2010). Childhood development of common drive to a human leg muscle during ankle dorsiflexion and gait. Journal of Physiology, 588(Pt 22), 4387-4400.

Portellano, J. A. (2007). Neuropsicología infantil. Madrid: Síntesis.

Roncesvalles, M. N. C., Woollacott, M. H. y Jensen, J. L. (2001). Development of lower extremity kinetics for balance control in infants and young children. Journal of Motor Behavior, 33(2), 180-192. 
Schneider, K., Zernicke, R. F., Ulrich, B. D., Jensen, J. L. y Thelen, E. (1990). Understanding movement control in infants through the analysis of limb intersegmental dynamics. Journal of Motor Behavior, 22(4), 493-520.

Shumway-Cook, A. y Woollacott, M. H. (2007). Motor Control: Translating Research Into Clinical Practice. Philadelphia: Lippincott Williams y Wilkins.

Sundermier, L., Woollacott, M. H., Roncesvalles, N. y Jensen, J. (2001). The development of balance control in children: comparisons of EMG and kinetic variables and chronological and developmental groupings. Experimental Brain Research, $136,340-350$.

Thelen, E. (1995). Motor development. A new synthesis. American Psychologist, 50(2), 79-95.

Thelen, E. y Cooke, D. W. (1987). Relationship between newborn stepping and later walking: A new interpretation. Developmental Medicine \& Child Neurology, 29(3), 380-393.

Thelen, E., Kelso, J. A. S. y Fogel, A. (1987). Selforganizing systems and infant motor development. Developmental Review, 7, 39-65.

Thelen, E., Schöner, G., Scheier, C. y Smith, L. B. (2001). The dynamics of embodyment: a field theory of infant perseverative reaching. Journal of Behavioral and Brain Science, 24(1), 1-34.

Thelen, E., Ulrich, B. D. y Niles, D. (1987). Bilateral coordination in human infants: stepping on a split-belt treadmill. Journal of Experimental Psychology: Human Perception and Performance, 13(3), 405-410.

Williams, C. M., Tinley, P. y Curtin, M. (2010). Idiopathic toe walking and sensory processing disfunction. Journal of Foot and Ankle Research, 3, 16-20.

Yang, J. F., Lam, T., Pang, M. Y. C., Lamont, E., Musselman, K. y Seinen, E. (2004). Infant stepping: a window to the behaviour of the human pattern generator for walking. Canadian Journal of Physiology andPharmacology, 82, 662-674.

Zelazo, P. R. (1983). The development of walking: New findings and old assumptions. Journal of Motor Behavior, 15(2), 99-137.

Zelazo, P. R. y Leonard, E. L. (1983). The dawn of active thought. In K. W. Fisher (Ed.), Levels and Transitions in Children's Development (pp. 37-50). San Francisco: Jossey-Bass.

Zwart, R., Ledebt, A., Fong, B. F., De Vries, H. y Savelsbergh, G. J. P. (2005). The affordance of gap crossing in toddlers. Infant Behavior \& Development, 28, 145-154. 